\title{
Time Domain simulation of PD Propagation in XLPE Cables Considering Frequency Dependent Parameters
}

\author{
Wei Zhang ${ }^{\mathrm{a}}$, Jian $\mathrm{He}^{\mathrm{b}}$, Lin Tian ${ }^{\mathrm{b}}$, Xuejun $\mathrm{Lv}^{\mathrm{b}}$, Hong-Jie $\mathrm{Li}^{\mathrm{a}}{ }^{*}$ and Chongxin Liu ${ }^{\mathrm{a}}$ \\ ${ }^{a}$ Xi'an Jiaotong University, No.28 Xianning West Road, Xi'an, 710049, China \\ ${ }^{b}$ Linyi Power Supply Ltd., $\quad$ No.130 Jinque Hill Road, Linyi, 276000, China
}

\begin{abstract}
Partial discharge (PD) detection and location is of great significance for the power cable insulation condition monitoring, where analysis of the PD propagation is needed. This paper presents the single-core model of cross-linked polyethylene (XLPE) cable considering the influence of skin effect and semi-conducting screens. To analysis the propagation of PD pulse in time domain, the model firstly is rational approximated using vector fitting method. Based on the fitting results, the $\pi$ section lumped parameters model is then proposed. Examples on two cables with different permittivity approximating forms of semiconducting screens are presented.
\end{abstract}

Keywords: Semi-conducting screen, state-space model, vector fitting (VF), XLPE cable

\section{Introduction}

The semi-conducting screens can have significant impact on high frequency wave propagation in power cables [1], [2]. The high frequency cable model that can be used over $100 \mathrm{MHz}$ is firstly described in [1], and more accurate model for extruded cables is developed in [3]. A few works on the characterization of semi-conducting layers in cables are carried out, and the complex permittivity of the dispersive layers is usually represented by Cole-Cole and Debye models [4], [5]. Although there is good performance between the high frequency cable model and the actual measurements, it is still a tough problem to solve these models in time domain.

Vector fitting (VF) is a robust numerical method which can be applied to fitting of measured or calculated frequency responses with rational function approximations [6]. This methodology has captured increasing interest in transient electromagnetic analysis and its fitting model may be easily represented by equivalent circuit with passive elements [7].

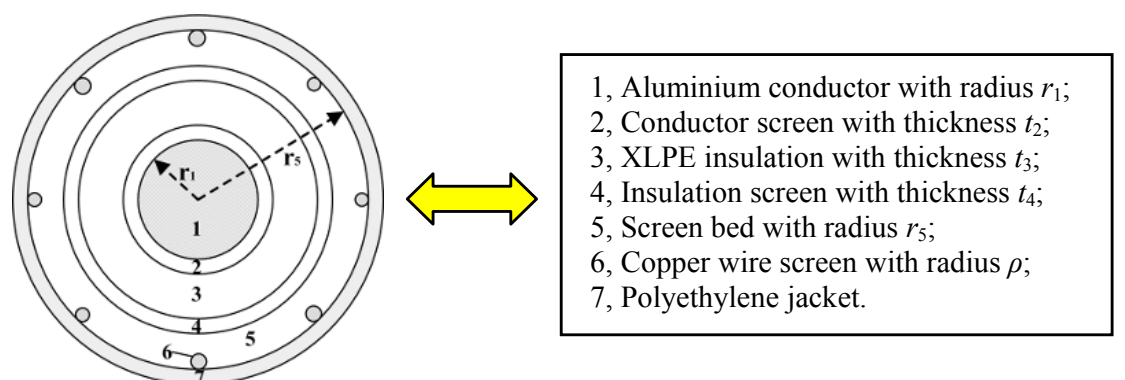

Fig.1. Geometry of XLPE cable.

\footnotetext{
* Manuscript received June 13, 2012; revised August 14, 2012.

Corresponding author. Tel.: +86-29-8266 4123; fax: +86-29-8266 8626; E-mail address: hjli@mail.xjtu.edu.cn.
} 
This paper introduces the VF fitting model into the time domain analysis of single-core power cable models considering the influence of both skin effect and semi-conducting screens. Through which, the generic $\pi$ circuit section of the power cable is proposed. Using the data from [4] and [5], high frequency pulse propagation is simulated by state-space method.

\section{Power Cable Model}

The single-core XLPE cable to be studied is shown in Fig.1, and its equivalent circuit model per unit length (p.u.1.) consist of series impedance $Z(\omega)$ and shunt admittance $Y(\omega)$ are given as follows [3],[4].

\subsection{Series impedance $Z(\omega)$}

Considering the skin effect in the conductor and metallic screen, the series impedance $Z(\omega)$ is expressed by:

$$
Z(\omega)=\frac{1}{2 \pi r_{1}} \sqrt{\frac{j \omega \mu_{0}}{\sigma_{1}}}+\frac{j \omega \mu_{0}}{2 \pi} \ln \frac{r_{5}}{r_{1}}+\frac{1}{2 \pi \rho n} \sqrt{\frac{j \omega \mu_{0}}{\sigma_{6}}}
$$

where $\sigma_{1}$ and $\sigma_{6}$ are the conductivities of aluminum and copper respectively, $\mu_{0}$ is the permeability of free space, $n$ is the total number of copper wires in the metallic screen.

\subsection{Shunt admittance $Y(\omega)$}

The semi-conducting screens and XLPE insulation contribute to the shunt admittance $Y(\omega)$ of the extruded cable, which is given by:

$$
Y(\omega)=\frac{1}{\sum_{i=2}^{5} \frac{1}{Y_{i}}}
$$

and

$$
Y_{i}=j \omega \frac{2 \pi \varepsilon_{0} \varepsilon_{i}^{*}}{\ln \left(r_{i} / r_{i-1}\right)}
$$

where $\varepsilon_{0}$ is the permittivity of free space, $r_{i}$ and $r_{i-1}$ are the outer and inner radius of layer $i$ respectively, and $\varepsilon_{i}^{*}=\varepsilon_{i}^{\prime}-j \varepsilon_{i}^{\prime \prime}$ is the complex permittivity for layer $i$, and for the XLPE insulation: $\varepsilon^{\prime}=2.3$ and $\varepsilon^{\prime \prime}=0.001$.

In addition, the complex permittivity of the semi-conducting screens obtained through measurements is described in [4], which is represented by two Cole-Cole functions and some attached terms, whereas the complex permittivity of the dispersive layers is described by Debye model in [5]. No matter what form of the complex permittivity in the cable model is, it is hard to analysis in time domain.

\section{Fitting Model and Equivalent Circuits}

Assume that function $F(\omega)$ represents the frequency response of a frequency dependent system, which can be rational approximated in the following form:

$$
F_{f i t}(\omega)=\sum_{i=1}^{N} \frac{c_{i}}{j \omega-a_{i}}+j \omega h+d=j \omega\left(\sum_{i=1}^{M} \frac{r_{i}}{j \omega-p_{i}}+h\right)+d
$$


where $r_{i}$ and $p_{i}$ are residues and poles which may be either real or conjugate complex numbers, $h$ and $d$ are real. All the coefficients in equation (4) can be calculated by the VF procedure described in [6].

Once the fitting models are obtained, they can be easily associated with equivalent circuits with passive elements. Reference [7] shows the equivalent circuit of the p.u.l. series impedance of a lossy transmission line. Similarly, we can obtain the equivalent circuit of the p.u.l. shunt admittance of the semi-conducting layers. To facilitate the analysis, the total admittance $Y_{s c}(\omega)$ of the semiconductor layers is modeled. $Y_{s c}(\omega)$ has the same form as (3) not including the XLPE part.

Applying the VF method for $Z(\omega)$ and $Y_{s c}(\omega)$, they will be fitted in the form of (4), and their corresponding equivalent models are shown in Fig.2. In which, Fig.2(a) represents the equivalent model of $Z(\omega)$ and $R_{0}=d, L_{0}=h, R_{i}=r_{i}, L_{i}=-r_{i} / p_{i},(i=1-N)$, while admittance $Y_{s c}(\omega)$ is represented in Fig.2(b) and $G_{0}=d, C_{0}=h, G_{i}=r_{i},(i=1-M)$. Moreover, it should be noted that $R_{0}$ and $G_{0}$ are the corresponding parameters in the DC conditions $(\omega=0)$ for $Z(\omega)$ and $Y_{s c}(\omega)$, respectively.

\section{State Space Model of Power Cables}

Based on the equivalent circuit in Fig.2, the generic $k$-th $\pi$ circuit section of power cable is represented in Fig.3. In which, $i_{\mathrm{k} \theta}$ and $i_{(k+1) 0}$ are currents flowing through $Z(\omega)$ in the $k$-th and $(k+1)$-th $\pi$ circuits, whereas $v_{k 0}$ and $v_{(k+1) 0}$ are the voltage across $Y(\omega) ; G_{0}$ and $C_{0}$ are the conductance and capacitance of the XLPE insulation, respectively.

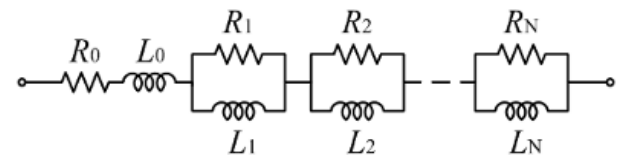

(a)

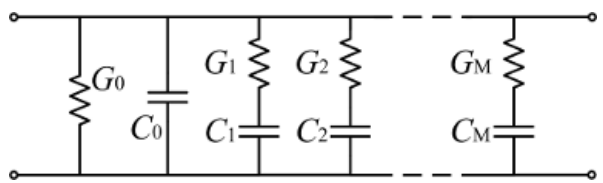

(b)

Fig.2. Equivalent circuits of fitting model for power cable. (a) Series impedance; (b) Admittance of semi-conducting layers.

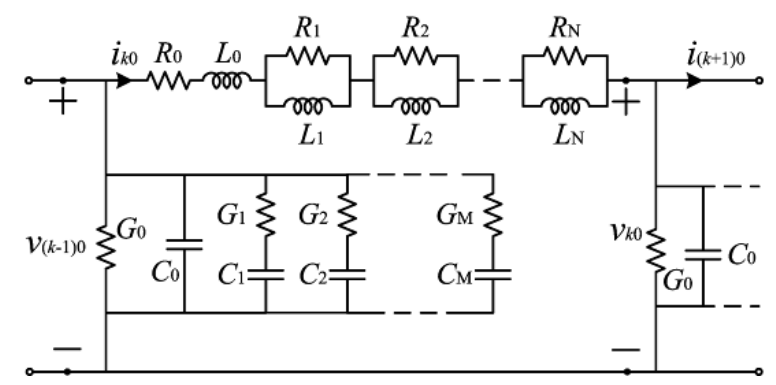

Fig.3. Equivalent $\pi$ circuit for power cable.

Assume that $i_{k 0}, i_{k 1}, i_{k 2}, \ldots, i_{k N}$ are currents through $L_{0}, L_{1}, L_{2}, \ldots, L_{N}$, and $v_{k 1}, v_{k 2}, \ldots$, $v_{k M}$ are voltages across $C_{1}, C_{2}, \ldots, C_{M}$, respectively. Considering $K \pi$ sections connected in cascade, the state differential equations in time domain can be written as:

$$
\dot{\mathbf{X}}=\mathbf{A X}+\mathbf{B} v_{s}
$$

where $\mathbf{X}$ and $\mathbf{B}$ are vectors with $(M+N+2) K$ dimensions, and $\mathbf{A}$ is $(M+N+2) K \times(M+N+2) K$ dimensional matrix, $v_{s}$ is excitation source, their specific forms are as follows:

$$
\begin{aligned}
& \mathbf{X}^{\top}=\left[\begin{array}{llll}
\mathbf{X}_{1} & \mathbf{X}_{2} & \cdots & \mathbf{X}_{K}
\end{array}\right] \\
& \mathbf{B}^{\top}=\left[\begin{array}{llll}
1 / L_{0} & 0 & 0 & \cdots
\end{array}\right]
\end{aligned}
$$




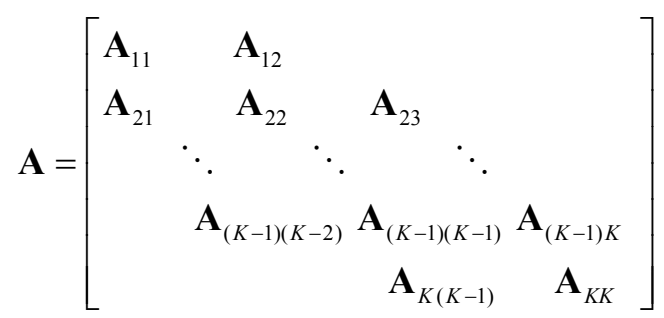

And for the $k$-th $\pi$ section, the related parameters are:

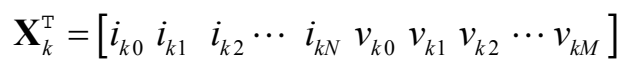

The generic submatrix $\mathbf{A}_{k k}$ with $(\mathrm{M}+\mathrm{N}+2)$ dimensions is expressed by:

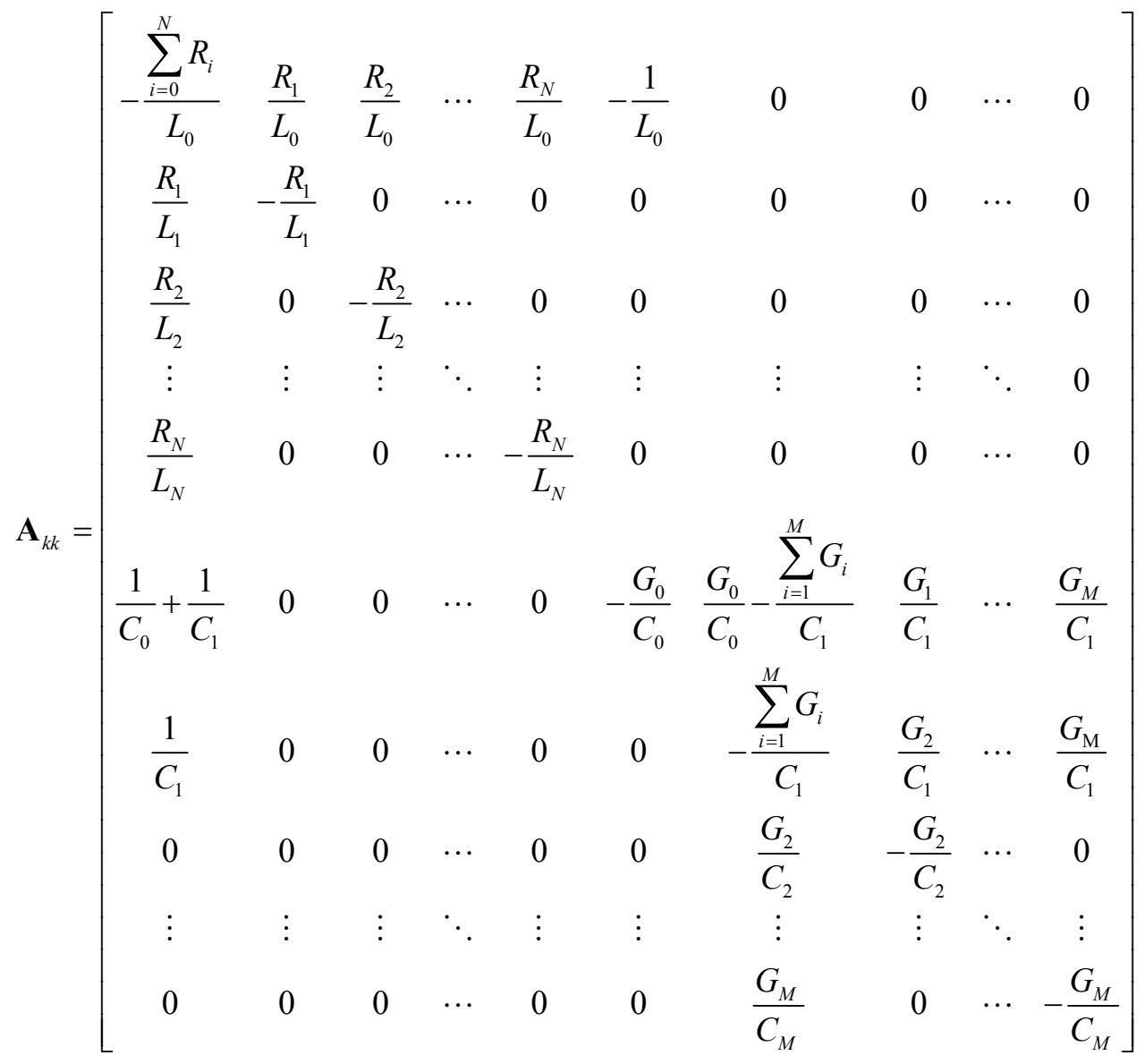

$\mathbf{A}_{k(k-1)}$ and $\mathbf{A}_{(k-1) k}$ having the same dimensions as $\mathbf{A}_{k k}$ are very highly sparse, and there are only a few non-zero elements in each of them, where:

$$
\left\{\begin{array}{l}
\mathbf{A}_{\mathrm{k}(\mathrm{k}-1)}(1,(N+2) K)=\frac{1}{L_{0}} \\
\mathbf{A}_{(\mathrm{k}-1) \mathrm{k}}((N+2) K, 1)=-\left(\frac{1}{C_{0}}+\frac{1}{C_{1}}\right), \quad \mathbf{A}_{(\mathrm{k}-1) \mathrm{k}}((N+2) K, 1)=-\frac{1}{C_{1}}
\end{array}\right.
$$

The state equations (5) are first-order linear ordinary differential equations and they can be easily solved with numerical methods. In this paper, Runge-Kutta method was used to solve the state space model. 


\section{Calculation Examples and Discussion}

This section presents example for the purpose of applying the proposed model. The XLPE cables to be studied are from the medium voltage cable called cable 1 in [4] (MVC) and the high voltage cable in [5] (HVC). The complex permittivity forms of which are Cole-Cole and Debye models, respectively. The details of the cables investigated are in Table 1.

Table 1. Cable parameters used in calculation (All units are in $\mathrm{mm}$ ).

\begin{tabular}{lcc}
\hline Parameter & MVC & HVC \\
\hline Conductor radius $\left(r_{1}\right)$ & 7.3 & 15 \\
Conductor screen thickness $\left(t_{2}\right)$ & 0.6 & 1.3 \\
Insulation thickness $\left(t_{3}\right)$ & 5.5 & 18 \\
Insulation screen thickness $\left(t_{4}\right)$ & 0.4 & 1.3 \\
Screen bed radius $\left(r_{5}\right)$ & 15.8 & 35.6 \\
\hline
\end{tabular}

\subsection{Modelling results by vector fitting}

In the rational approximation of the series impedance $Z(\omega)$ and the total admittance $Y_{s c}(\omega)$ of the semiconductor layers for both MVC and HVC, vector fitting procedure was implemented in the range of $0.01 \mathrm{~Hz}$ to $100 \mathrm{MHz}$. Fig. 4 shows the fitting results after 20 iterations using 2 pairs of complex starting poles. It is seen that very good approximations have been achieved. The maximum of the root-mean-square (RMS) errors was found to be $1.88 \mathrm{E}-8$.

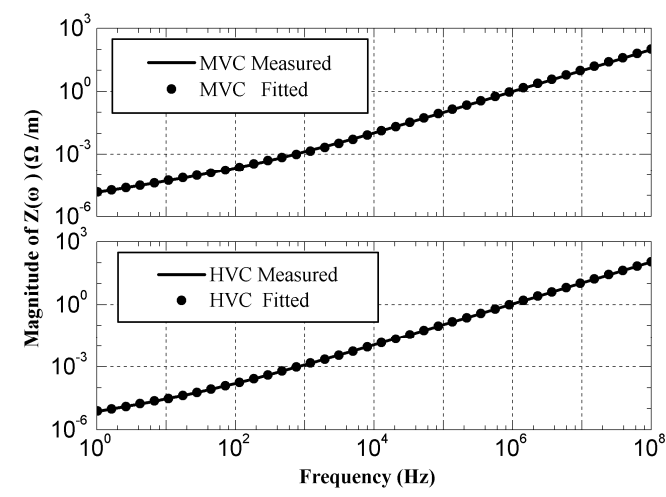

(a)

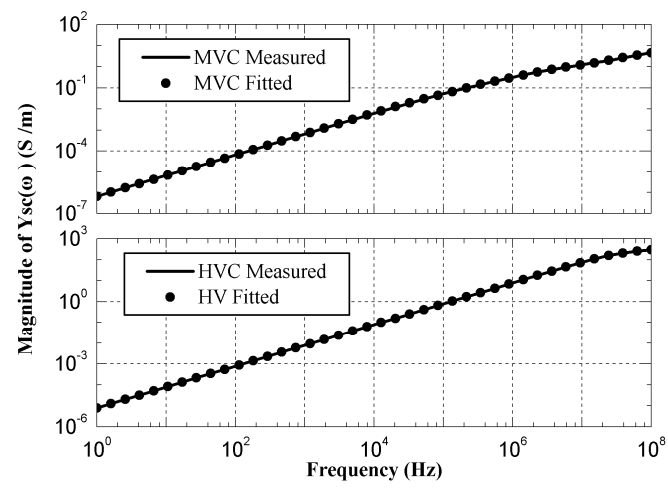

(b)

Fig.4. Vector Fitting results of MVC and HVC. (a) Magnitude of $Z(\omega)$ p.u.1.; (b) Magnitude of $Y_{s c}(\omega)$ p.u.1..

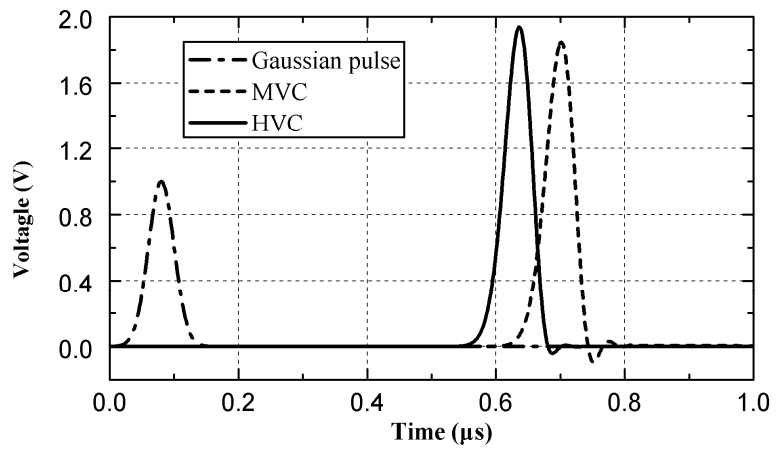

Fig.5. Equivalent $\pi$ circuit for power cable. 
Table 2. Fitting Parameters of Series Impedance Shown in Fig.3.

\begin{tabular}{cccccc}
\hline \multicolumn{3}{c}{ Resistance $(\Omega / \mathrm{m})$} & \multicolumn{3}{c}{ Inductance $(\mathrm{H} / \mathrm{m})$} \\
\hline Parameter & MVC & HVC & Parameter & MVC & HVC \\
\hline R0 & $9.7332 \times 10^{-4}$ & $3.9298 \times 10^{-5}$ & L0 & $1.6261 \times 10^{-7}$ & $1.7527 \times 10^{-7}$ \\
R1 & $3.4701 \times 10^{-4}$ & $2.9315 \times 10^{-4}$ & L1 & $1.2757 \times 10^{-7}$ & $3.7109 \times 10^{-8}$ \\
R2 & $5.0486 \times 10^{-5}$ & $4.2661 \times 10^{-5}$ & L2 & $3.2066 \times 10^{-7}$ & $1.0949 \times 10^{-7}$ \\
R3 & $1.4774 \times 10^{-5}$ & $1.1480 \times 10^{-5}$ & L3 & $7.5397 \times 10^{-7}$ & $3.1659 \times 10^{-7}$ \\
R4 & $8.9996 \times 10^{-6}$ & $5.3953 \times 10^{-6}$ & L4 & $4.7701 \times 10^{-6}$ & $1.9469 \times 10^{-6}$ \\
\hline
\end{tabular}

Table 3. Fitting Parameters of Shunt Admittance Shown in Fig.3

\begin{tabular}{cccccc}
\hline \multicolumn{3}{c}{ Conductance $(\mathrm{S} / \mathrm{m})$} & & Capacitance & $(\mathrm{F} / \mathrm{m})$ \\
\hline Parameter & MVC & HVC & Parameter & MVC & HVC \\
\hline G0 & $1.0514 \times 10^{-13}$ & $7.4673 \times 10^{-13}$ & C0 & $2.4182 \times 10^{-10}$ & $1.7175 \times 10^{-10}$ \\
\hline G1 & $1.1599 \times 10^{2}$ & $5.2223 \times 10^{1}$ & C1 & $4.8327 \times 10^{-9}$ & $2.3088 \times 10^{-7}$ \\
\hline G2 & $5.9236 \times 10^{-9}$ & $4.0945 \times 10^{-21}$ & C2 & $1.9717 \times 10^{0}$ & $1.3118 \times 10^{-10}$ \\
\hline G3 & $2.5524 \times 10^{-8}$ & $1.0159 \times 10^{-6}$ & C3 & $7.0381 \times 10^{-1}$ & $2.2574 \times 10^{2}$ \\
\hline G4 & $3.5475 \times 10^{-8}$ & $3.6614 \times 10^{-22}$ & C4 & $1.3123 \times 10^{-1}$ & $1.0983 \times 10^{-14}$ \\
\hline G5 & $3.1862 \times 10^{-8}$ & $3.1824 \times 10^{-23}$ & C5 & $1.1743 \times 10^{-2}$ & $2.9021 \times 10^{-20}$ \\
\hline
\end{tabular}

Also, the frequency dependent parameters in Fig.3 $(M=N=4)$ are given in Table 2 and Table 3 in Appendix.

\subsection{Transient simulation}

In the following calculations, the length of the extruded power cables is 100 meters. Determining the number of $\pi$ sections is important in the transient analysis of system represented by lumped-parameter $\pi$ circuits connected in cascades. In this paper, the statistical correlation method was adopted which was implemented by the following procedure.

Step 1) Choose $K_{0}$ as the initial number of $\pi$ sections.

Step 2) Calculate the voltages $V_{1}$ and $V_{2}$ at the observation points with $K_{0}$ and $K_{0}+\Delta K \pi$ sections considered, respectively.

Step 3) Compute the Pearson correlation coefficient $C_{0}$ of $V_{1}$ and $V_{2}$. If $C_{0}$ is greater than a setting value, accept $K_{0}$ as the final result; otherwise set $K_{0}=K_{0}+\Delta K$, go to Step 2.

Based on the method above, the cable was finally divided into $100 \pi$ circuits connected in cascades. Simulation was carried out between 0 to $1 \mu \mathrm{s}$ with $1 \mathrm{~ns}$ as the calculation step. Gaussian pulse with frequency width of $20 \mathrm{MHz}$ is injected into the XLPE cables, and the open-circuit voltages at the receiving end are shown in Fig.5. From which we can obtain the propagation velocities of MVC and HVC are about $162 \mathrm{~m} / \mu \mathrm{s}$ and $179 \mathrm{~m} / \mu \mathrm{s}$, respectively. Furthermore, there is higher attenuation in MVC for the same injected pulse.

\section{Conclusion}

The characteristics of frequency dependent parameters in power cables can be efficiently rational approximated by VF method. Based on the equivalent circuits proposed in this paper, it is easy to obtain the state space model in time domain for extruded cable models considering skin effects and semi-conducting screens.

\section{References}

[1] Stone G, Boggs S. Propagation of partial discharge pulses in shielded power cable. In: Proc. of IEEE Conference on Electrical Insulation and Dielectric Phenomena, 1982:275-280. 
[2] Weeks W, Diao YM. Wave propagation characteristics in underground power cable. IEEE Trans. on Power Apparatus and Systems, 1984; 103(10):2816-2826.

[3] Mugala G, Eriksson R, Pettersson P. Dependence of XLPE insulated power cable wave propagation characteristics on design parameters. IEEE Trans. on Dielectrics and Electrical Insulation, 2007; 14(2):393-399.

[4] Mugala G, Eriksson R, Gafvert U, Petterson P. Measurement technique for high frequency characterization of semiconducting materials in extruded cables. IEEE Trans. on Dielectrics and Electrical Insulation, 2004; 11(3):471-480.

[5] Jobava R, Heinrich R, Pommerenke D, Kalkner W, Gheonjian A. Efficient FDTD simulation of fields in coaxial cables with multi-layered insulation partially formed by dispersive layers of extremely high permittivity. In: Proc. of the 7 International Seminar/Workshop on Direct and Inverse Problems of Electromagnetic and Acoustic Wave Theory, 2002:91-94.

[6] Gustavsen B, Semlyen A. Rational approximation of frequency domain responses by vector fitting. IEEE Trans. on Power Delivery, 1999; 14(3):052-1061.

[7] Sarto M, Scarlatti A, Holloway C. On the use of fitting models for the time-domain analysis of problems with frequency-dependent parameters. In: Proc. of 2001 IEEE EMC International Symposium, 2001:588-593. 